\title{
Ballistic transport through graphene nanostructures of velocity and potential barriers
}

Q.1 P M Krstajic ${ }^{1}$ and P Vasilopoulos ${ }^{2}$

\author{
${ }^{1}$ Institute of Microelectronic Technologies and Single Crystals (IHTM), Belgrade University, \\ Njegoševa 12, 11000 Belgrade, Serbia \\ ${ }^{2}$ Department of Physics, Concordia University, 7141 Sherbrooke Ouest Montréal, \\ QC, H4B 1R6, Canada
}

Received 23 January 2011, in final form 20 February 2011

Published

Online at stacks.iop.org/JPhysCM/23/000000

\begin{abstract}
We investigate the electronic properties of graphene nanostructures when the Fermi velocity and the electrostatic potential vary in space. First, we consider the transmission $T$ and conductance $G$ through single and double barriers. We show that $G$ for velocity barriers differs markedly from that for potential barriers for energies below the height of the latter and it exhibits periodic oscillations as a function of the energy for strong velocity modulation. Special attention is given to superlattices (SLs). It is shown that an applied bias can efficiently widen or shrink the allowed minibands of velocity-modulated SLs. The spectrum in the Kronig-Penney limit is periodic in the strength of the barriers. Collimation of an electron beam incident on an SL with velocity and potential barriers is present but it disappears when the potential barriers are absent. The number of additional Dirac points may change considerably if barriers and wells have sufficiently different Fermi velocities.
\end{abstract}

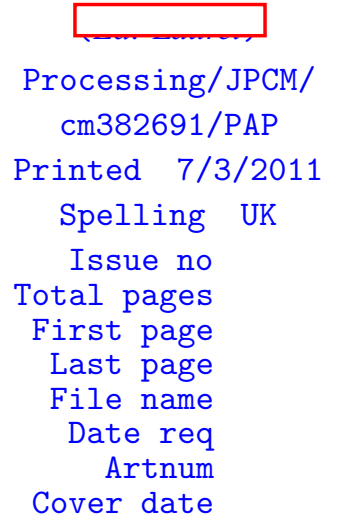

\section{Introduction}

Graphene is a monolayer of carbon atoms arranged on a hexagonal lattice and was first synthesized in 2004 [1] despite early theoretical works that doubted its existence. Since then it has been the subject of intense study and research made by both theoretical and experimental condensed matter physicists. The unique property of charge carriers in graphene is that they behave like relativistic massless, chiral fermions with the 'speed of light' replaced by the Fermi velocity. This brings about many unusual phenomena such as an anomalous quantum Hall effect [2, 3], Klein tunnelling [4], Zitterbewegung [5], etc. All these phenomena have been studied extensively. Another reason for such a high interest in this material is that nanostructures based on graphene may find applications in nanoelectronics [5], as sensitive sensors of magnetic fields and even of a gas [6]. Already exploiting the submicron long mean-free paths in graphene-based devices has led to the production of transistors [7].

Recently, there has been interest in velocity-modulated structures $[8,9]$ of graphene where the Fermi velocity of charge carriers is made to vary in space [10] by appropriate doping [11], by placing a grounded metal plane close to graphene [8], or by imprinting on it a lateral superlattice with hexagonal symmetry [12]. In this work we investigate in more detail ballistic transport not only through simple velocity barriers $[8,9]$ but also through more complex nanostructures, such as double barriers and superlattices (SLs) with both velocity $v(\mathbf{r})$ and/or potential $V(\mathbf{r})$ variation in space that, to our knowledge, has not been considered in the literature. We find that the conductance of strongly modulated velocity barriers oscillates periodically with the energy and differs markedly from that for potential barriers and energies below the height of the latter. In addition, we consider the collimation of an electron beam incident on such SLs and the emergence of extra Dirac points. In doing so we make contact with the corresponding results for the usual SLs without velocity modulation [13-17] and show that if the potential barriers are absent, there is neither modulation nor additional Dirac points but both reappear when such barriers are present.

The paper is organized as follows. In section 2 we present the theoretical basis needed to study the transport properties of 
velocity-modulated structures made of graphene. In section 2 the model is used to evaluate the transmission through and conductance of a single and double velocity barrier. Section 3 is devoted to SLs with periodic variation in the Fermi velocity and barrier heights. The last section summarizes our results and conclusions.

\section{Theoretical framework}

We consider a graphene sheet in which the electron velocity varies in space, that is $v=v(\mathbf{r})$. The relevant Hamiltonian, which allows for a space-dependent velocity, is $[8,10]$

$$
\hat{H}=-\mathrm{i} \hbar \sqrt{v(\mathbf{r})} \vec{\sigma} \cdot \nabla_{\mathbf{r}} \sqrt{v(\mathbf{r})} .
$$

Here $\vec{\sigma}=\left(\sigma_{x}, \sigma_{y}\right)$ is a two-dimensional (2D) Pauli matrix, and $\Psi=\left(\psi_{\mathrm{A}}, \psi_{\mathrm{B}}\right)^{\mathrm{T}}$ is a two component spinor. In obtaining equation (1) it is assumed that the variation of the velocity is slow enough on the scale of the lattice constant. It is convenient to introduce the auxiliary spinor $\Phi(\mathbf{r})=\sqrt{v(\mathbf{r})} \Psi(\mathbf{r})$, which leads to the following eigenvalue problem

$$
\hat{H} \Psi(\mathbf{r})=-\mathrm{i} \hbar v(\mathbf{r}) \vec{\sigma} \cdot \nabla_{\mathbf{r}} \Phi(\mathbf{r})=E \Phi(\mathbf{r}),
$$

which is the familiar Dirac-like equation for usual graphene [5]. The boundary matching conditions can be inferred from the continuity equation $\operatorname{div} \mathbf{J}(\mathbf{r})=0$, where the probability current $\mathbf{J}(\mathbf{r})$ is given by

$$
\mathbf{J}(\mathbf{r})=v(\mathbf{r}) \Psi^{\dagger}(\mathbf{r}) \vec{\sigma} \Psi(\mathbf{r})
$$

However, we consider only piece-wise variations of $v$ along the $x$ axis, $v=v(x)$. This leads to the boundary matching condition

$$
\sqrt{v_{1}} \Psi\left(x=0_{-}\right)=\sqrt{v_{2}} \Psi\left(x=0_{+}\right),
$$

for $v\left(0_{-}\right)=v_{1}$ and $v\left(0_{+}\right)=v_{2}$.

The theoretical model will be first applied to the problem of tunnelling through a single velocity barrier. Consider a barrier of width $D$, made of two different slabs of graphene (see figure 1(a)) such that the Fermi velocity is given by

$$
v(x)= \begin{cases}v_{1}, & x<0, \\ v_{2}, & 0 \leqslant x \leqslant D, \\ v_{1}, & x>D .\end{cases}
$$

We denote by $\theta_{1}$ the angle of incidence on the barrier, by $\theta_{2}$ the angle inside the barrier, and by $k_{x}$ and $q_{x}$ the wavevectors inside and outside the velocity barrier. The transmission $T=$ $|t|^{2}$ in terms of the angles $\theta_{1}$ and $\theta_{2}$ reads [8]

$$
\begin{aligned}
& T^{(\mathrm{vel})}\left(\theta_{1}\right) \\
& =\frac{\cos ^{2} \theta_{1}\left[1-\xi^{2} \sin ^{2} \theta_{1}\right]}{\cos ^{2} \theta_{1}\left[1-\xi^{2} \sin ^{2} \theta_{1}\right]+(1-\xi)^{2} \sin ^{2} \theta_{1} \sin ^{2}\left(q_{x} D\right)} .
\end{aligned}
$$

The relation between the angles $\theta_{1}$ and $\theta_{2}$ can be found from the conservation of energy and of the wavevector $k_{y}$ (due to translational invariance along the $y$ axis) as

$$
\frac{\sin \theta_{1}}{\sin \theta_{2}}=\frac{v_{1}}{v_{2}}=\frac{1}{\xi} \text {. }
$$
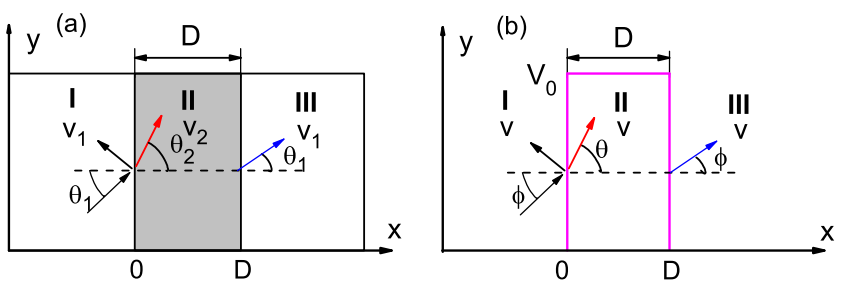

Figure 1. Scheme of a velocity barrier (a) made of two graphene slabs with different velocities $v_{1}$ and $v_{2}$, and (b) a usual potential barrier with height $V_{0}$ and $v=v_{\mathrm{F}}$ everywhere the same.

This relation is in fact a quantum mechanical version of Snell's law in optics. For $\xi>1$ one may define the critical angle $\theta_{1 \mathrm{c}}^{(\mathrm{vel})}=\arcsin (1 / \xi)$ above which $\left(\theta_{1} \geqslant \theta_{1 \mathrm{c}}\right)$ the transmission is negligible as the wavevector $q_{x}$ is then imaginary. Actually, the transmission is exactly zero for $\theta_{1}=\theta_{1 \mathrm{c}}$ as can be seen from equation (6). For $\theta_{1}>\theta_{1 \mathrm{c}}$, equation (7) should be modified to account for evanescent states

$$
\begin{aligned}
& T^{\text {(vel) }}\left(\theta_{1}\right) \\
& =\frac{\cos ^{2} \theta_{1}\left[\xi^{2} \sin ^{2} \theta_{1}-1\right]}{\cos ^{2} \theta_{1}\left[\xi^{2} \sin ^{2} \theta_{1}-1\right]+(1-\xi)^{2} \sin ^{2}\left(\theta_{1}\right) \sinh ^{2}\left(\kappa_{x} D\right)},
\end{aligned}
$$

where $\kappa_{x}=\mathrm{i} q_{x}=\left[k_{y}^{2}-\left(E_{\mathrm{F}} /\left(\hbar v_{2}\right)\right)^{2}\right]^{1 / 2}$. The critical angle can also be defined for a standard potential barrier [18] by the same argument. It is given by

$$
\theta_{1 \mathrm{c}}^{(\mathrm{pot})}=\arcsin \left(\left|E_{\mathrm{F}}-V_{0}\right| /\left|E_{\mathrm{F}}\right|\right) .
$$

The values of $q_{x}$ and $k_{x}$ are given by

$$
q_{x}=\left[\left(E_{\mathrm{F}} / \hbar v_{2}\right)^{2}-k_{y}^{2}\right]^{1 / 2}, \quad k_{x}=\left[\left(E_{\mathrm{F}} / \hbar v_{1}\right)^{2}-k_{y}^{2}\right]^{1 / 2} .
$$

Notice that equation (6) gives $T=1$ for $\theta_{1}=0$ regardless of the values of the other parameters which is another manifestation of the Klein paradox in tunnelling, this time through a velocity barrier. The transmission reaches unity also for $q_{x} D=n \pi$, with $n$ integer and $q_{x}$ given by equation (10), as in the standard case [19] in which $q_{x}=\left[\left(\left(E_{\mathrm{F}}-V_{0}\right) / \hbar v_{2}\right)^{2}-\right.$ $\left.k_{y}^{2}\right]^{1 / 2}$

In figure 2 we plot the transmission through a barrier of thickness $D=100 \mathrm{~nm}$ as a function of the angle of incidence $\theta_{1}$ for $E_{\mathrm{F}}=50 \mathrm{meV}$ and four different values of $\xi: \xi=0.2$ (solid, black curve), $\xi=0.5$ (dashed, blue curve), $\xi=1.2$ Q.3 (dotted, red curve) and $\xi=1.5$ (dash-dotted, green curve). The Fermi velocity outside the barrier is kept constant at the value $v_{1}=0.7 \times 10^{6} \mathrm{~m} \mathrm{~s}^{-1}$. Note that the transmission is virtually zero for $\xi>1$ (last two curves) and $\theta_{1}>\theta_{1 \mathrm{c}}$. The transmission is not exactly zero for $\theta_{1}>\theta_{1 \mathrm{c}}$ but it is very small due to the evanescent nature of the states in the barrier.

It is interesting to compare equation (6) with the formula for the transmission through a potential barrier in a graphene which reads [5]

$$
\begin{aligned}
& T^{(\text {pot })}\left(\theta_{1}\right) \\
& =\frac{\cos ^{2} \theta_{1} \cos ^{2} \theta_{2}}{\left[\cos \theta_{1} \cos \theta_{2} \cos \left(q_{x} D\right)\right]^{2}+\left(1-s s^{\prime} \sin \theta_{1} \sin \theta_{2}\right)^{2} \sin ^{2}\left(q_{x} D\right)} .
\end{aligned}
$$




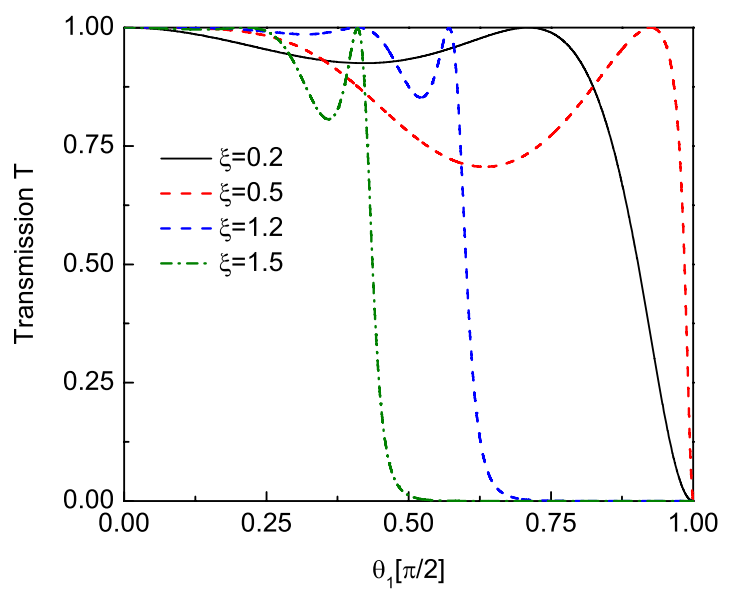

Figure 2. Transmission $T$ through a velocity barrier versus the angle of incidence $\theta_{1}$ for four different values of the velocity ratio of $\xi$ $\left(=v_{2} / v_{1}\right), \xi=0.2,0.5,1.2$, and 1.5 .

Here $s=\operatorname{sgn}\left(E_{\mathrm{F}}\right)$ and $s=\operatorname{sgn}\left(E_{\mathrm{F}}-V_{0}\right)$. It is possible to make a direct connection between equations (11) and (6) when they are expressed in terms of the angles only. There is one small point, here one should calculate the wavevectors using the velocities of the corresponding materials. In equation (11) both $s$ and $s^{\prime}$ are +1 because there are no potential barriers, and it is assumed $E_{\mathrm{F}}>0$. Inserting $\xi$ from equation (7) into equation (6), one obtains that $T^{(\mathrm{pot})}\left(\theta_{1}\right)=T^{(\mathrm{vel})}\left(\theta_{1}\right)$ are given by the same expression but with the wavevectors $q_{x}$ defined appropriately in each case.

At this point one may also define a critical angle, for total reflection, if both velocity and potential barriers are present. Following the same procedure gives

$$
\theta_{1 \mathrm{c}}^{(v p)}=\arcsin \left(\frac{v_{1}\left|E_{\mathrm{F}}-V_{2}\right|}{v_{2}\left|E_{\mathrm{F}}-V_{1}\right|}\right) .
$$

The material denoted by ' 2 ' acts as a barrier, so that usually $V_{1}=0$ and $V_{2}=V_{0}$. Note that the critical angle when there is only velocity modulation, $\theta_{1 \mathrm{c}}^{(\mathrm{vel})}$, does not depend on the energy. In order to illustrate the dependence of the transmission on both the angle of incidence and the energy, one may construct a contour plot of $T$ versus the momentum components $k_{x}$ and $k_{y}$. In figure 3 we show such contour plots for two different values of $\xi$ : (a) $\xi=0.2$ (upper panel) and $\xi=1.2$ (lower panel). For $\xi=0.2<1$ one sees the oscillations near $k_{x} \rightarrow 0$, which are absent for $\xi=1.2$, that are due to small $\sinh ^{2}\left(\kappa_{x} D\right)$ in the latter. In both panels the transmission is equal to 1 for small $k_{y} \rightarrow 0$, which is a manifestation of Klein tunnelling.

Given the transmission amplitude one may calculate the conductance, which is a measurable quantity. For small values of an applied source-to-drain bias and very low temperatures the conductance is given by

$$
G=G_{0} \int_{-\pi / 2}^{\pi / 2} T\left(E_{\mathrm{F}}, \theta_{1}\right) \cos \theta_{1} \mathrm{~d} \theta_{1},
$$

with $G_{0}=2 e^{2} E_{\mathrm{F}} L_{y} / h^{2} v_{\mathrm{F}}$ and $L_{y}$ the length of the slab in the $y$ direction. The factor $\cos \theta_{1}$ comes from the probability current density $j=v_{\mathrm{F}} \Psi^{\dagger} \sigma_{x} \Psi \propto v_{\mathrm{F}} \cos \theta_{1}$.
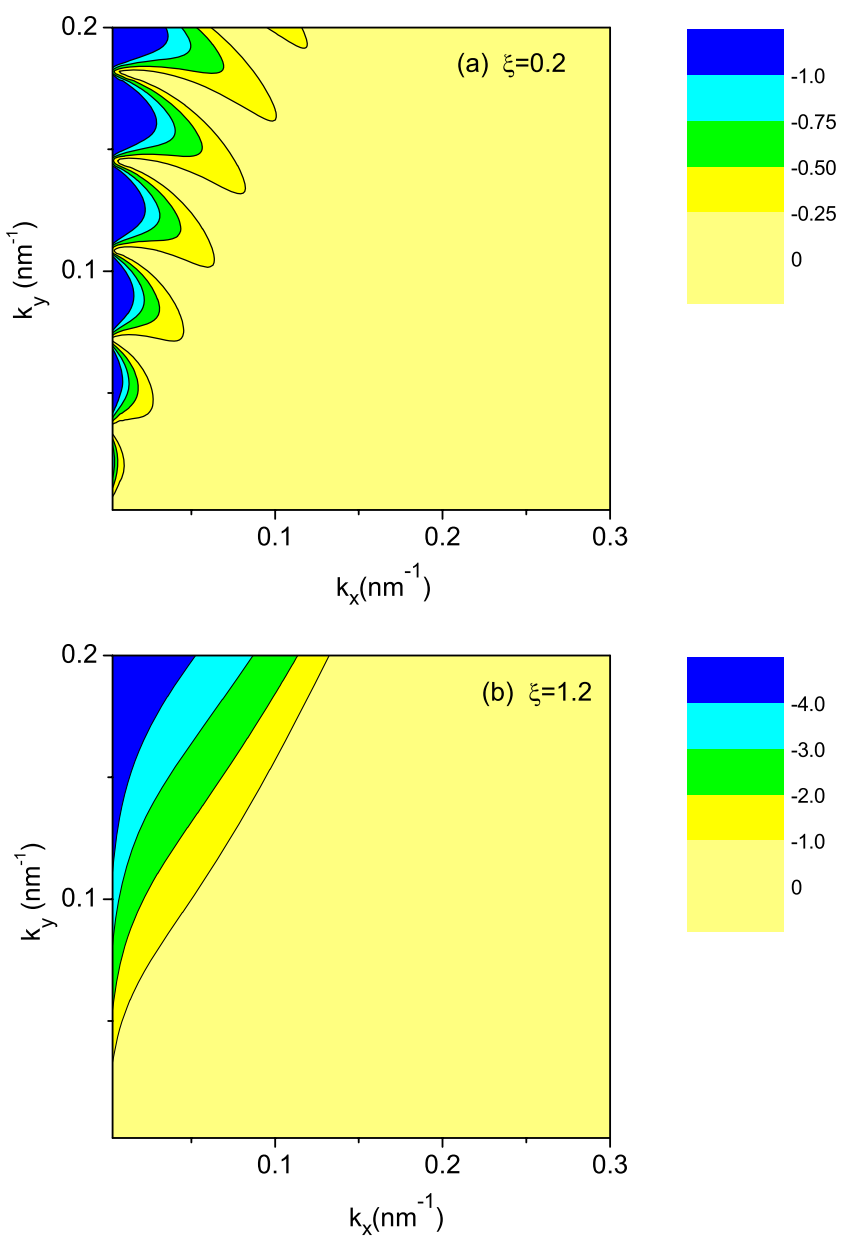

Figure 3. Contour plots of the transmission versus momentum components $k_{x}$ and $k_{y}$ for two different values of the parameter $\xi$ : (a) $\xi=0.2$ and (b) $\xi=1.2$. Notice the logarithmic scale in the colour meter.

In figure 4 we plot the conductance through a single velocity barrier versus the Fermi energy for four different velocity ratios $\xi=0.2,0.5,1.2$, and 1.5 . The barrier width is $D=100 \mathrm{~nm}$, the velocity $v_{1}=0.7 \times 10^{6} \mathrm{~m} \mathrm{~s}^{-1}, a=1.42 \AA$ the lattice constant, and $L_{y}=100 \sqrt{3} a$. The inset shows the conductance through a potential barrier of the same width and height $V_{0}=100 \mathrm{meV}$ but for $v_{\mathrm{F}}=10^{6} \mathrm{~m} \mathrm{~s}^{-1}$ everywhere, i.e. for $\xi=1$. The minimum near $V_{0}=100 \mathrm{meV}$ is because the real wavevector $q_{x}$ vanishes at this value, so almost does the transmission, and correspondingly the conductance.

For $\xi<1$ weak oscillations appear (dashed curve) that completely disappear for $\xi \geqslant 1$. However, for very small $\xi \ll 1$ we see regular oscillations, as the energy increases (solid curve) that are in sharp contrast with those through the usual potential barriers, see inset, as long as the energy is less than the barrier height [20]. One further sees, upon contrasting the solid and dashed curves, that these oscillations depend on $\xi$.

The oscillations for $\xi \ll 1$ can be understood as follows. For $\xi \ll 1$ we may neglect the term $-\xi^{2} \sin \theta_{1}$ in equation (6) and neglect the dependence of $q_{x}$ on $\theta_{1}$ since $v_{2} \ll v_{1}$. This 


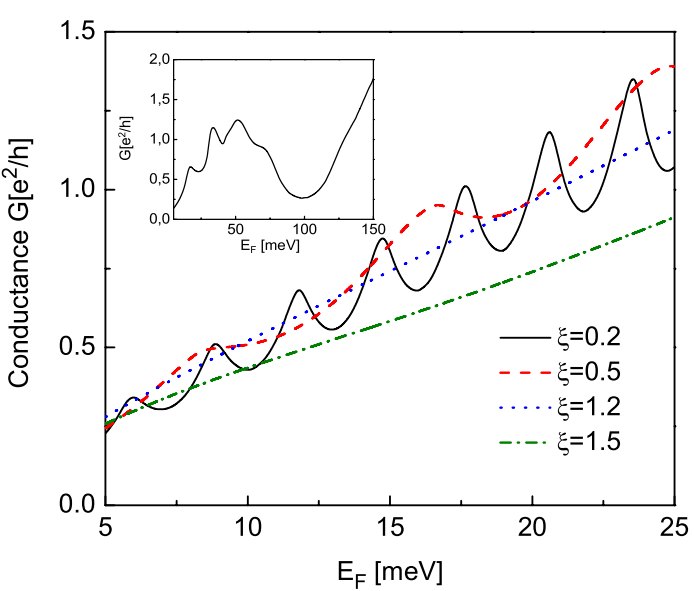

Figure 4. Conductance $G$ through a velocity barrier as a function of the Fermi energy $E_{\mathrm{F}}$ for four different values of the velocity ratio $\xi$ $\left(=v_{2} / v_{1}\right), \xi=0.2,0.5,1.2$, and 1.5. Inset: $G$ through a single potential barrier with $V_{0}=100 \mathrm{meV}$ and $v_{\mathrm{F}}=10^{6} \mathrm{~m} \mathrm{~s}^{-1}$ everywhere $(\xi=1)$.

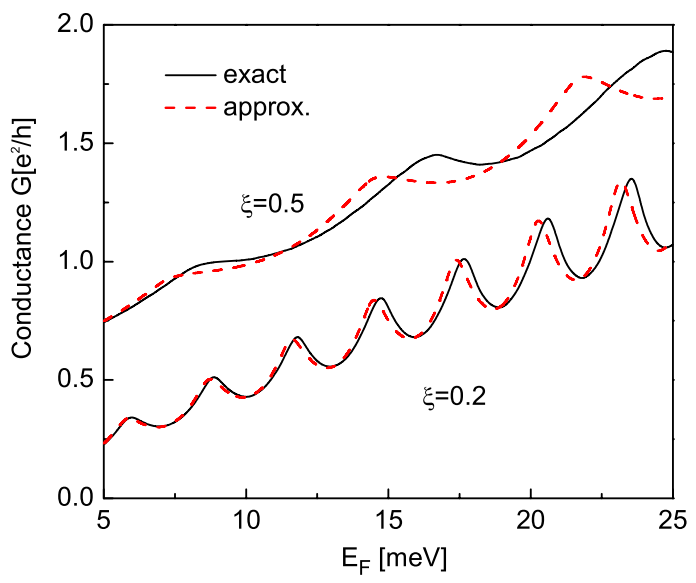

Figure 5. Comparison between the exact values of $G$ (solid, black curves) and the approximate (dashed, red curves), as given in equation (14), for a single velocity barrier and two values of $\xi: 0.2$ and 0.5 . For clarity the curves for $\xi=0.5$ are shifted up by $0.5 e^{2} / h$.

leads to the approximate formula for the conductivity

$$
G=\frac{G_{0}}{1-A}\left(2-\frac{A}{\sqrt{1-A}} \ln \frac{1+\sqrt{1-A}}{1-\sqrt{1-A}}\right)
$$

where $A=(1-\xi)^{2} \sin ^{2}\left(E_{\mathrm{F}} D / \hbar v_{1}\right)$. In figure 5 we compare $G$, given by equation (14), with the exact numerically evaluated one of a velocity barrier for two values of $\xi: 0.2$ and 0.5 . For clarity the curves for $\xi=0.5$ are shifted up by $0.5 e^{2} / h$. The agreement is quite satisfactory for $\xi=0.2$ and the oscillations are well reproduced.

In figure 6 we show the conductance through a double barrier, with the same barrier parameters as in figure 4 , and an inter-barrier distance equal to the barrier widths $d=D=$ $100 \mathrm{~nm}$. For the sake of comparison, we show in the inset the conductance through two potential barriers of the same width and height $V_{0}=100 \mathrm{meV}$, while $v_{\mathrm{F}}=10^{6} \mathrm{~m} \mathrm{~s}^{-1}$ everywhere, i.e. for $\xi=1$. Notice again the near-vanishing of

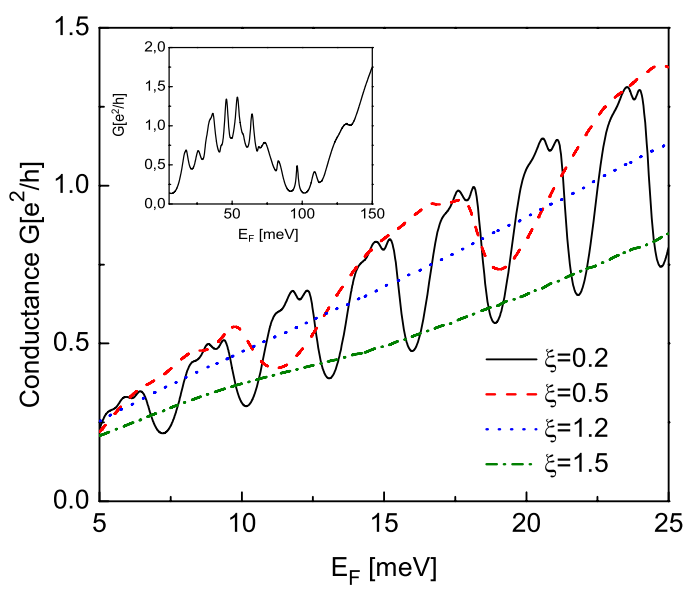

Figure 6. Conductance $G$ through a double velocity barrier, as a function of the Fermi energy $E_{\mathrm{F}}$, for four different values of the velocity ratio $\xi\left(=v_{2} / v_{1}\right), \xi=0.2,0.5,1.2$, and 1.5 . The inter-barrier distance is $d=100 \mathrm{~nm}$. Inset: $G$ through a double potential barrier with $V_{0}=100 \mathrm{meV}$ and $\xi=1\left(v_{\mathrm{F}}=10^{6} \mathrm{~m} \mathrm{~s}^{-1}\right)$.

the transmission for $V_{0}=100 \mathrm{meV}$. The order of magnitude of $G$ is the same as for a single barrier, but, as expected, the local maxima/minima for $\xi=0.2$ and 0.5 are more pronounced since the resonances in the transmission are sharper.

\section{Velocity- and potential-barrier superlattices}

\subsection{Dispersion relation}

Now we turn our attention to an SL with varying velocity and height of the potential barriers. We assume that the SL period $d$ is the same in both cases and that the widths of the regions of different potentials coincide with those of different velocities. Of course, one may choose, for instance, the zero potential to be in one or the other material (with different $v$ ). Let us suppose that such an SL is made of two graphene slabs $\mathrm{A}$ and $\mathrm{B}$, of widths $w_{\mathrm{a}}$ and $w_{\mathrm{b}}$, with velocities $v_{\mathrm{a}}, v_{\mathrm{b}}$, and electrostatic potentials $V_{\mathrm{A}}, V_{\mathrm{B}}$, respectively. If in each constituent layer the electrostatic potential and velocity are constant, $V(x)=V_{i}, v(x)=v_{i}$, the wavefunction is a sum of two terms corresponding to the two directions

$$
\Psi_{i}(\mathbf{r})=a_{i} \sqrt{v_{i}}\left(\begin{array}{c}
1 \\
s_{i} \mathrm{e}^{\mathrm{i} \theta_{i}}
\end{array}\right) \mathrm{e}^{\mathrm{i} q_{x i} x}+b_{i} \sqrt{v_{i}}\left(\begin{array}{c}
1 \\
-s_{i} \mathrm{e}^{-\mathrm{i} \theta_{i}}
\end{array}\right) \mathrm{e}^{-\mathrm{i} q_{x i} x},
$$

where $s_{i}=\operatorname{sgn}\left(E-V_{i}\right)$ and $\tan \theta_{i}=q_{x i} / k_{y}$. Starting from equations (4) and (15), one may construct a $2 \times 2$ boundary matching matrix in the $i$ th layer that connects the coefficients $\left(a_{i}, b_{i}\right)$ on both sides of this interface by the matrix

$$
M_{i}=\sqrt{v_{i}}\left(\begin{array}{cc}
1 & 1 \\
s_{i} \mathrm{e}^{\mathrm{i} \theta_{i}} & -s_{i} \mathrm{e}^{-\mathrm{i} \theta_{i}}
\end{array}\right)
$$

where $s_{i}=\operatorname{sgn}\left(E-V_{i}\right)$. The propagation matrix $P_{i}$ is the same as in the standard case; with $w_{i}$ the width of the $i$ th layer it reads

$$
P_{i}=\left(\begin{array}{cc}
\mathrm{e}^{\mathrm{i} q_{i} w_{i}} & 0 \\
0 & \mathrm{e}^{-\mathrm{i} q_{i} w_{i}}
\end{array}\right)
$$


In each layer $i$ the relevant wavevectors $q_{\mathrm{a}}$ and $q_{\mathrm{b}}$ are given by

$$
\begin{aligned}
& q_{\mathrm{a}}=\left[\left[\left(E_{\mathrm{F}}-V_{\mathrm{A}}\right) / \hbar v_{\mathrm{a}}\right]^{2}-k_{y}^{2}\right]^{1 / 2}, \\
& q_{\mathrm{b}}=\left[\left[\left(E_{\mathrm{F}}-V_{\mathrm{B}}\right) / \hbar v_{\mathrm{b}}\right]^{2}-k_{y}^{2}\right]^{1 / 2} .
\end{aligned}
$$

The transfer matrix $T_{m}$ connects two points one period apart, e.g. points 1 and 2 in figure 7 , in the manner

$$
\left(\begin{array}{l}
a_{2} \\
b_{2}
\end{array}\right)=T_{m}\left(\begin{array}{l}
a_{1} \\
b_{1}
\end{array}\right) .
$$

On the other hand, Bloch's theorem applied to an SL with a period $d=w_{\mathrm{a}}+w_{\mathrm{b}}$ requires

$$
\left(\begin{array}{l}
a_{2} \\
b_{2}
\end{array}\right)=\exp \left(\mathrm{i} \beta_{x} d\right)\left(\begin{array}{l}
a_{1} \\
b_{1}
\end{array}\right)
$$

where $\beta_{x}$ is an additional quantum number that stems from the periodicity of the SL. From equations (19) and (20) one sees that $\exp \left(\mathrm{i} \beta_{x} d\right)$ is one of the eigenvalues of the transfer matrix $T_{m}$, the other being its complex conjugate $\exp \left(-\mathrm{i} \beta_{x} d\right)$. One easily arrives at the dispersion relation [19]

$$
\cos \left(\beta_{x} d\right)=\operatorname{Tr} T_{m} / 2,
$$

which determines the band structure of the SL. Now the transfer matrix $T_{m}$ is given in terms of the propagation matrix $P_{i}$ and the boundary matching matrix $M_{i}$ by

$$
T_{m}=P_{\mathrm{A}} M_{\mathrm{A}}^{-1} M_{\mathrm{B}} P_{\mathrm{B}} M_{\mathrm{B}}^{-1} M_{\mathrm{A}},
$$

where $P_{i}$ and $M_{i}$ are defined in equations (16) and (17). Explicit calculation of the trace of the transfer matrix leads to

$$
\begin{aligned}
& \cos \left(\beta_{x} d\right)=\cos \left(q_{\mathrm{a}} w_{\mathrm{a}}\right) \cos \left(q_{\mathrm{b}} w_{\mathrm{b}}\right) \\
& \quad-\frac{s_{\mathrm{A}} s_{\mathrm{B}}-\sin \theta_{\mathrm{A}} \sin \theta_{\mathrm{B}}}{\cos \theta_{\mathrm{A}} \cos \theta_{\mathrm{B}}} \sin \left(q_{\mathrm{a}} w_{\mathrm{a}}\right) \sin \left(q_{\mathrm{b}} w_{\mathrm{b}}\right) .
\end{aligned}
$$

Note that equation (23) is valid for all values of energies due to the factors $s_{\mathrm{A}}$ and $s_{\mathrm{B}}$, as long as $q_{i}$ are real. For large transverse wavevectors $k_{y}, q_{i}$ may become purely imaginary and the cosine and sine functions should be replaced by the hyperbolic functions. The allowed minibands satisfy the condition $\left|\cos \left(\beta_{x} d\right)\right| \leqslant 1$ and their widths increase with energy. The dispersion relation (23) is identical to the one obtained for an SL of piece-wise constant potential barriers [21] $V(x)$. (Reference [4] considered the more general case with a nonzero mass term, $m>0$.) This can be explained by the fact that the new boundary matching matrices are the same as the old ones simply multiplied by $\sqrt{v_{i}}$ but this factor cancels out when constructing the transfer matrix. Alternatively, one may use the auxiliary spinor $\Phi(\mathbf{r})=$ $\sqrt{v(\mathbf{r})} \Psi(\mathbf{r})$ from the outset.

It is interesting to take the Kronig-Penney limit of equation (23) expressed by $w_{\mathrm{b}} \rightarrow 0$ and $V_{\mathrm{B}} \rightarrow \infty$ but in such a way that the product $V_{\mathrm{B}} w_{\mathrm{b}}$ is kept constant $V_{\mathrm{B}} w_{\mathrm{b}}=\hbar v_{\mathrm{F}} \Omega_{\mathrm{B}}$ with $\Omega_{\mathrm{B}}$ a dimensionless quantity. Then $s_{\mathrm{B}}=-1, \theta_{\mathrm{B}} \rightarrow 0$ $\left(q_{\mathrm{b}} \gg k_{y}\right)$, and equation (23) turns into

$$
\begin{aligned}
& \cos \left(\beta_{x} \Lambda\right)=\cos \left(\Omega_{\mathrm{B}}\right) \cos \left(q_{\mathrm{a}} w_{\mathrm{a}}\right) \\
& \quad+\sin \left(\Omega_{\mathrm{B}}\right) \sin \left(q_{\mathrm{a}} w_{\mathrm{a}}\right) / s_{\mathrm{A}} \cos \theta_{\mathrm{A}} \cos \theta_{\mathrm{B}} .
\end{aligned}
$$

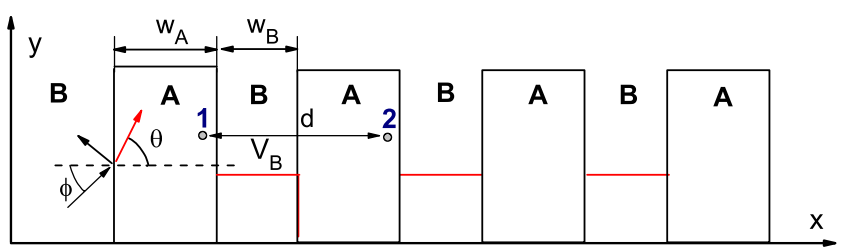

Figure 7. Scheme of a superlattice made by variation of both velocity $(v)$ and potentials $V(x)$ (red lines). It is assumed that $V_{\mathrm{A}}=0$.

Equation (24) shows that in this limit the spectrum is a periodic function of the strength of the barriers $\propto \Omega_{\mathrm{B}}$ in agreement with [22]. Notice though that this limit can probably be achieved only if the potential is present, since there is a limitation in making the ratio $v_{\mathrm{b}} / v_{\mathrm{a}}$ high.

Returning to equation (23) one may investigate at what (nonzero) values of the potentials $V_{\mathrm{A}}$ and $V_{\mathrm{B}}$ the forbidden minibands disappear. This may happen for $\theta_{\mathrm{A}}=0$ or normal incidence. Then we have

$$
\cos \left(\beta_{x} d\right)=\cos \left(q_{\mathrm{a}} w_{\mathrm{a}} \pm q_{\mathrm{b}} w_{\mathrm{b}}\right),
$$

which always lies in the range $[-1,1]$. The condition $\theta_{\mathrm{A}} \approx$ $\theta_{\mathrm{B}} \approx 0$ may be approximately fulfilled if $q_{\mathrm{a}}=q_{\mathrm{b}}$ and $E \ll V_{\mathrm{A}}, V_{\mathrm{B}}$, which in turn entails

$$
\frac{E_{\mathrm{F}}-V_{\mathrm{A}}}{E_{\mathrm{F}}-V_{\mathrm{B}}}=\frac{v_{\mathrm{a}}}{v_{\mathrm{b}}} .
$$

Equation (26) may hold independent of the value of $E_{\mathrm{F}}$ if one considers the case when $E_{\mathrm{F}}$ is very small, in a certain range, compared to both $V_{\mathrm{A}}$ and $V_{\mathrm{B}}$, as already assumed. This leads to

$$
\frac{V_{\mathrm{A}}}{V_{\mathrm{B}}} \approx \frac{v_{\mathrm{a}}}{v_{\mathrm{b}}} .
$$

Let us assume, for instance, $0 \mathrm{meV} \leqslant E_{\mathrm{F}} \leqslant 30 \mathrm{meV}$, $V_{\mathrm{A}}=60 \mathrm{meV}, V_{\mathrm{B}}=120 \mathrm{meV}, v_{\mathrm{a}}=5 \times 10^{5} \mathrm{~m} \mathrm{~s}^{-1}$, and $v_{\mathrm{b}}=10^{6} \mathrm{~m} \mathrm{~s}^{-1}$. The transmission through a finite SL $\left(N=25, w_{\mathrm{a}}=w_{\mathrm{b}}=20 \mathrm{~nm}\right)$ having such a potential and velocity variation is shown in figure 8 by the solid, red curve. For comparison, we also show the results when only the velocity barriers (black curve) are present. Further, the dashed, red curve corresponds to the transmission through an infinite SL with both $v(x)$ and $V(x)$ varying. One can see that the first allowed miniband is extended when $V_{\mathrm{A}}$ and $V_{\mathrm{B}}$ are nonzero and $V_{\mathrm{A}} / V_{\mathrm{B}}=v_{\mathrm{a}} / v_{\mathrm{b}}$. This confirms the approximate relation (27) and opens the possibility to enhance or tune a velocity-modulated SL by applying a bias. This could also be important in the opposite situation when a usual graphenebased SL $(v(x)=$ const $)$ does not attain a sufficiently high and needed ratio $q_{\mathrm{a}} / q_{\mathrm{b}}$ due to possible limitations in the maximum allowed bias.

\subsection{Collimation, extra Dirac points}

The spectrum of a graphene-based SL exhibits [22] interesting properties. For instance, the spectrum of a one-dimensional SL can become highly anisotropic [13] for certain SL parameters. This anisotropy can be made large enough so that the energy 


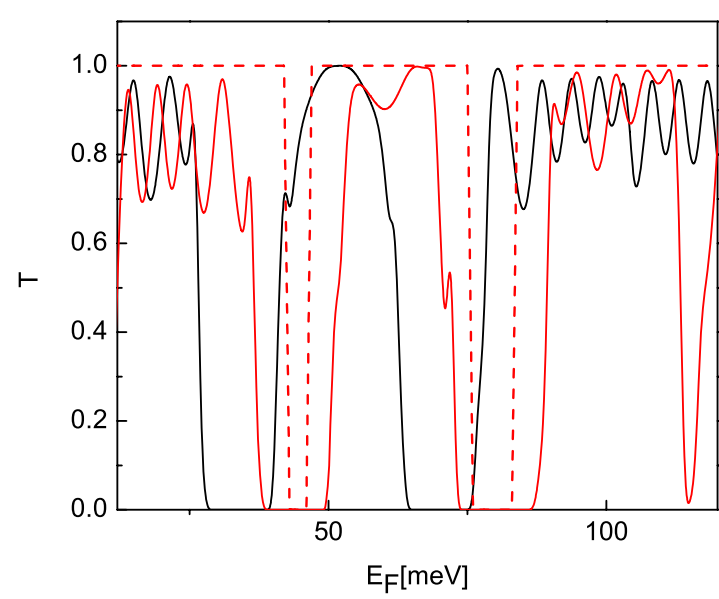

Figure 8. Transmission versus Fermi energy $E_{\mathrm{F}}$ in an SL with both velocity and potential barriers (red curve) and only velocity barriers (black curve). The number of units is $N=25$ and the other parameters $V_{\mathrm{A}}=60 \mathrm{meV}, V_{\mathrm{B}}=2 V_{\mathrm{A}}, v_{\mathrm{a}}=5 \times 10^{5} \mathrm{~m} \mathrm{~s}^{-1}$, and $v_{\mathrm{b}}=2 v_{\mathrm{a}}, w_{\mathrm{a}}=w_{\mathrm{b}}=20 \mathrm{~nm}$. The dashed, red curve shows the transmission of an infinite SL with both types of barriers.

spectrum is dispersionless in one direction [14]. This implies that an electron beam incident on an SL becomes collimated along the axis perpendicular to that direction. Further, if the parameters of SL are carefully tuned, additional Dirac points are possible $[22,16]$. Here we extend this analysis to a SL with spatial variation of both velocity and potential.

First, we begin by addressing the collimation of an electron beam, when the energy dispersion becomes approximately independent of $k_{y}$. It is proven that the collimation occurs in the vicinity of extra Dirac points $[22,17]$. Starting from equation (23) it is possible to extract the dispersion relation $E\left(\beta_{x}\right)$ with $k_{y}$ kept as a parameter. In panel (a) of figure 9 we show such an SL dispersion relation for three different values of $k_{y}: 0.0,0.2 \pi / d$, and $0.4 \pi / d$. The quantities are normalized such that $\epsilon=E d / \hbar v_{\mathrm{a}}, \beta_{x 0}=\beta_{x} d$, and $k_{y 0}=k_{y} d$, while the parameters of the SL are $\xi=0.9$, $V_{\mathrm{B}}=4 \pi \hbar v_{\mathrm{b}} / d, V_{\mathrm{A}}=0$, and $v_{\mathrm{a}}=10^{6} \mathrm{~m} \mathrm{~s}^{-1}$. For comparison in panel (b) we show this dispersion relation for bulk graphene, that is, with $V_{i}=0$ and $\xi=1$, when the SL quantum number $\beta_{x}$ becomes identical to $k_{x}$. From figure 9(a) it is obvious that the energy dispersion is independent of $k_{y}$ in a wide range of $\beta_{x 0}$, while in figure 9(b), for bulk graphene, it displays the expected dependence $\epsilon=\left[\beta_{x 0}^{2}+k_{y 0}^{2}\right]^{1 / 2}$.

It is important to emphasize that the collimation shown in figure 9(a) is present only when the potential barriers are present. For an SL with only the Fermi velocity $v(x)$ varying, the dispersion relation for the first miniband can be approximated by

$$
\varepsilon=\frac{2 \xi}{1+\xi}\left[\beta_{x 0}^{2}+k_{y 0}^{2}\right]^{1 / 2},
$$

for small energies $\varepsilon$ and $k_{y 0}$. The dependence on $k_{y 0}$ shows that indeed there is no collimation in such an SL.

We now turn to the additional Dirac points. One way to locate them is to assume some special values of the parameters [22] $\beta_{x}=0$ and $w_{\mathrm{a}}=w_{\mathrm{b}}$ (equal barrier and well
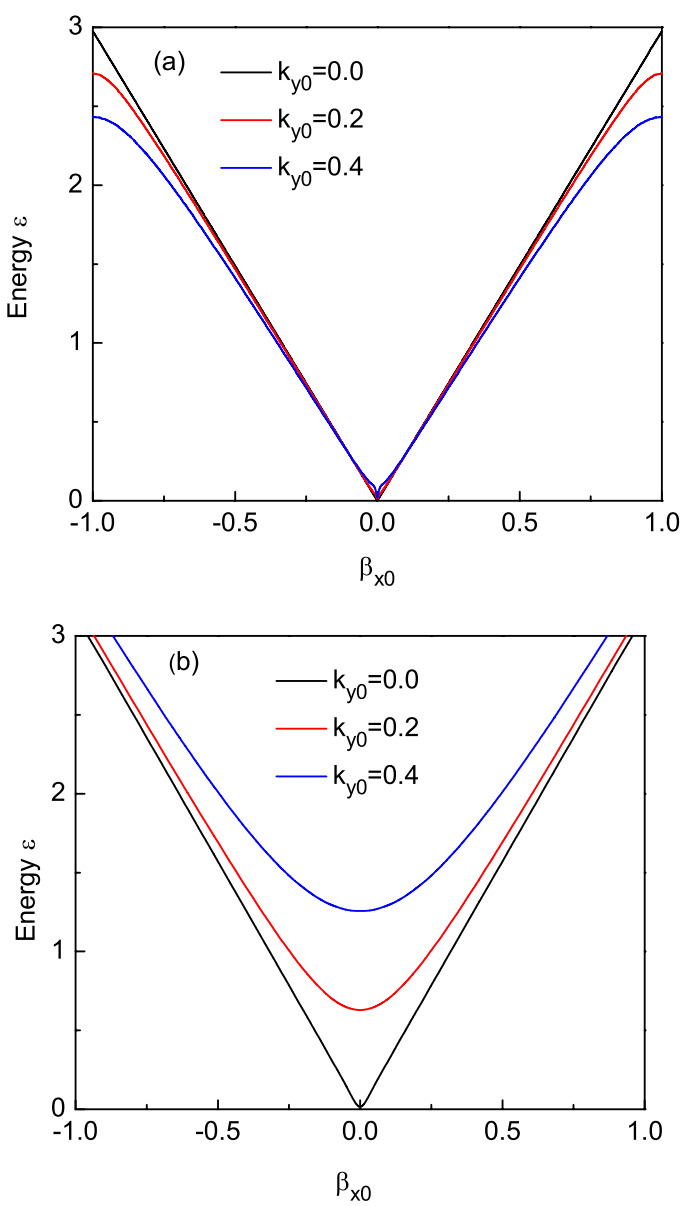

Figure 9. (a) Dispersion relation $\epsilon\left(\beta_{x 0}\right)$ of a velocity- and potential-modulated SL for three values of the wavevector $k_{y 0}: 0.0$ (black curve), 0.2 (red curve), and 0.4 (blue curve). The parameters are $\xi=0.9, V_{\mathrm{B}}=4 \pi \hbar v_{\mathrm{b}} / d\left(V_{\mathrm{A}}=0\right)$, and $v_{\mathrm{a}}=10^{6} \mathrm{~m} \mathrm{~s}^{-1}$. For comparison panel (b) shows this dispersion relation for bulk graphene $\left(\xi=1, V_{i}=0\right)$.

widths). It is assumed, as earlier, that $V_{\mathrm{A}}=0$. First one finds the energy $E_{e}$ around which the spectrum exhibits symmetry, that is when $q_{\mathrm{a}}=q_{\mathrm{b}}$ which yields $E_{e}=V_{\mathrm{B}} /(1+\xi)$. The dispersion relation becomes

$$
1=\cos ^{2} \kappa-\left[\left(\epsilon_{\mathrm{A}} \epsilon_{\mathrm{B}}-k_{y}^{2}\right) / q_{\mathrm{a}} q_{\mathrm{b}}\right] \sin ^{2} \kappa,
$$

where $\epsilon_{i}(i=\mathrm{A}, \mathrm{B})$ are the normalized energies $E / \hbar v_{i}$. One can readily see two sets of solutions: (1) for $k_{y}=0$ and (2) for $\kappa=j \pi(j=0,1,2, \ldots)$.

Case (1) $\left(k_{y}=0\right)$ corresponds to the main Dirac point and is not of interest here.

Case (2) leads to

$$
k_{y, j}= \pm\left[\left[\left(E_{e}-V_{\mathrm{B}}\right) / \hbar v_{\mathrm{b}}\right]^{2}-(2 j \pi / d)^{2}\right]^{1 / 2} .
$$

In figure 10 we show the dispersion curve $\epsilon\left(k_{y 0}\right)$ for an SL having equal barrier and well widths for three values of the parameter $\xi: 0.9,1.0$, and 1.1 . The wavevector $k_{y}$ and the energy are normalized $k_{y 0}=k_{y} d$ and $\epsilon=E d / \hbar v_{\mathrm{a}}$, while the reference level for the energy is $E_{e}=V_{\mathrm{B}} /(1+\xi)$ and varies with $\xi$. The Fermi velocity in the barrier is varied $v_{\mathrm{b}}=\xi v_{\mathrm{a}}$ 

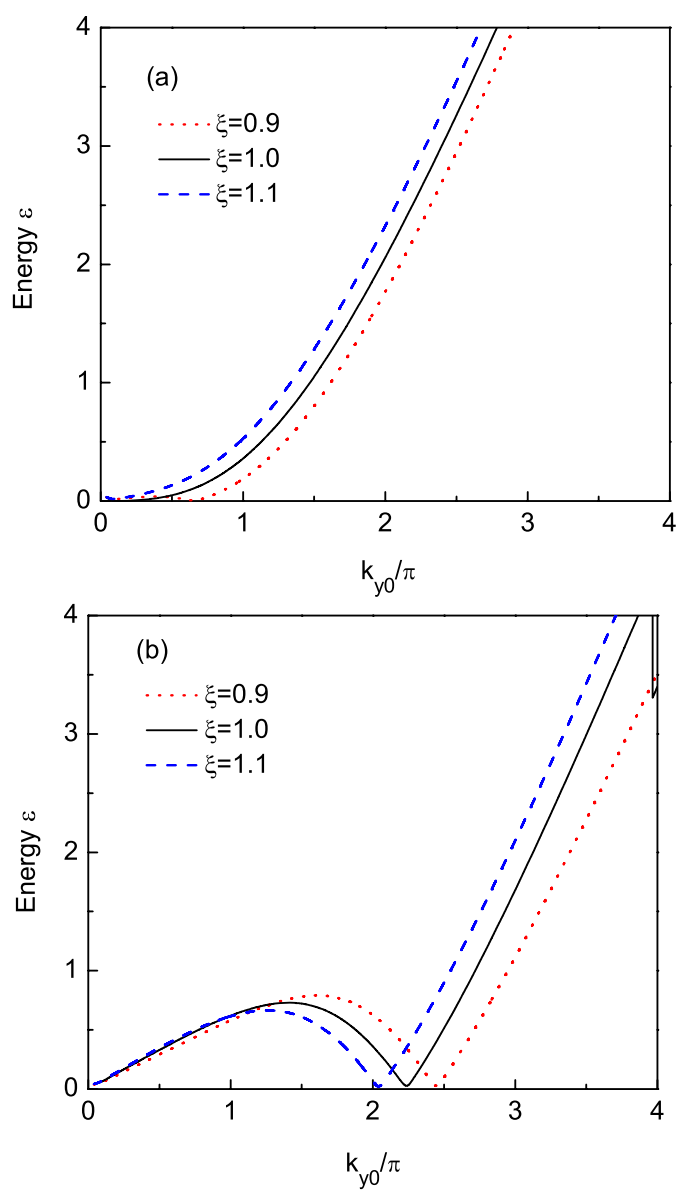

Figure 10. Dispersion relation $\epsilon\left(k_{y 0}\right)$ of velocity-and potential-modulated SLs for three values of the parameter $\xi: 0.9$ (dotted red curve), 1.0 (solid black curve), and 1.1 (dashed blue curve). Panel (a) corresponds to $V_{\mathrm{B}} / \hbar v_{\mathrm{b}}=4 \pi$ and panel (b) to $V_{\mathrm{B}} / \hbar v_{\mathrm{b}}=6 \pi$. The reference energy is that of the extra Dirac point located at $E_{e}=V_{\mathrm{B}} /(1+\xi)$ and all quantities are normalized.

with $v_{\mathrm{a}}=10^{6} \mathrm{~m} \mathrm{~s}^{-1}$, while $V_{\mathrm{A}}=0$ and $V_{\mathrm{B}}=6 \pi \hbar v_{\mathrm{F}} / d$. The second minima occur at values given by equation (30) for $j=1$. As seen in the figure, the difference in the curves is already visible for a relative change in the Fermi velocities of only $10 \%$.

To locate additional Dirac points for less trivial cases, i.e. for unequal barrier and well widths, one may assume $\cos \left(q_{\mathrm{a}} w_{\mathrm{a}}\right)= \pm 1$ and $\cos \left(q_{\mathrm{b}} w_{\mathrm{b}}\right)= \pm 1$ while the sine functions are zero. This is fulfilled only if $q_{\mathrm{a}} w_{\mathrm{a}}=j \pi$ and $q_{\mathrm{b}} w_{\mathrm{b}}=$ $(j+2 m) \pi$ where $j$ and $m$ are both integers. This in turn entails

$$
\begin{gathered}
{\left[E_{j, m} / \hbar v_{\mathrm{a}}\right]^{2}-k_{y}^{2}=\left[j \pi / w_{\mathrm{a}}\right]^{2},} \\
\left(\left(E_{j, m}-V_{\mathrm{B}}\right) / \hbar v_{\mathrm{b}}\right)^{2}-k_{y}^{2}=\left((j+2 m) \pi / w_{\mathrm{b}}\right)^{2} .
\end{gathered}
$$

Solving for the energy $E_{j, m}$, one arrives at a quadratic equation for $E_{j, m}$, whose solution is

$$
E_{j, m}=V_{\mathrm{B}}\left[-1 \pm \sqrt{1+\left(\xi^{2}-1\right) C}\right] /\left(\xi^{2}-1\right),
$$

with

$$
C=1+\left(\frac{\hbar v_{\mathrm{b}}}{V_{\mathrm{B}}}\right)^{2}\left[\left(\frac{j \pi}{w_{\mathrm{a}}}\right)^{2}-\left(\frac{(j+2 m) \pi}{w_{\mathrm{b}}}\right)^{2}\right]
$$

Having found $E_{j, m}$ the wavevector $k_{y j, m}$ can be obtained from equation (31); it is equal to

$$
k_{y j, m}= \pm\left[\left(E_{j, m} / \hbar v_{\mathrm{a}}\right)^{2}-\left(j \pi / w_{\mathrm{a}}\right)^{2}\right]^{1 / 2} .
$$

In the limit $\xi \rightarrow 1$ equation (33) has only one set of solutions $E_{j, m}=V_{\mathrm{B}} / 2 C$ and the result (B4) from [22] is obtained, while for $w_{\mathrm{a}}=w_{\mathrm{b}}$ and $m=0$ one recovers $E_{j, 0}=E_{e}$ and equation (35) turns into equation (30). According to equation (33), there are no extra Dirac points in the absence of potential barriers, i.e. for $V_{\mathrm{B}}=0$. Note that equation (33) is defined only for $C \geqslant-1 /\left(\xi^{2}-1\right)$, so that extra Dirac points do not exist for any combination of integers $j$ and $m$. This means that the number of Dirac points in a certain range of energies may change if the velocities $v_{\mathrm{a}}$ and $v_{\mathrm{b}}$ are sufficiently different. This is another difference with the standard case [22].

\section{Conclusions}

We investigated the electronic transport properties of graphene nanostructures with spatial variation of the Fermi velocity or/and of the potential. The conductance through a single and double velocity barrier exhibits a non-monotonic dependence on the Fermi energy $E_{\mathrm{F}}$ for $\xi<1\left(v_{2}<v_{1}\right)$ that is significantly different than that through the usual potential barriers for $E_{\mathrm{F}}$ smaller than their height. Depending on the value of $\xi<1$, one can see very well-pronounced oscillations in the conductance of single and double velocity barriers, see figures 4-6.

We also investigated the dispersion relation of SLs in which both $v_{\mathrm{F}}(x)$ and $V(x)$ vary in a piece-wise manner. It was shown that it is possible to tune the low-energy spectrum of a velocity-modulated SL by properly biasing it, that is, by superimposing a usual potential SL, with the same period. An example is the widening of the minibands inferred from that of the corresponding transmission shown in figure 8 . Electron collimation is present in such SLs but disappears if the usual potential barriers are absent. Extra Dirac points have been found in some cases and their number may change considerably if the difference in the Fermi velocity of the constituent materials is sufficiently high. These extra Dirac points disappear if the usual potential barriers are absent.

\section{Acknowledgment}

Our work was supported by the Canadian NSERC Grant No. OGP0121756.

\section{References}

[1] Novoselov K S, Geim A K, Morozov S V, Jiang D, Zhang Y, Dubonos S V, Grigorieva I V and Firsov A A 2004 Science 306666

[2] Novoselov K S, Geim A K, Morozov S V, Jiang D, Katsnelson M I, Grigorieva I V, Dubonos S V and Firsov A A 2005 Nature 438197

[3] Zhang Y, Tan Y-W, Stormer H L and Kim P 2005 Nature 438201

[4] Barbier M, Peeters F M, Vasilopoulos P and Pereira J M Jr 2008 Phys. Rev. B 77115446

[5] Neto A H C, Guinea F, Peres N M R, Novoselov K S and Geim A K 2009 Rev. Mod. Phys. 81109 
[6] Leenaerts O, Partoens B and Peeters F M 2008 Appl. Phys. Lett. 93193107

[7] Ponomarenko L A, Schedin F, Katsnelson M I, Yang R, Hill E W, Novoselov K S and Geim A K 2008 Science 320356

Stampfer C, Schurtenberger E, Molitor F, Guttinger J, Ihn T and Ensslin K 2009 Nano Lett. 82378

[8] Raoux A, Polini M, Asgari R, Hamilton A R, Fazio R and MacDonald A H 2010 Phys. Rev. B 81073407

[9] Concha A and Tešanović Z 2010 Phys. Rev. B 82033413

[10] Peres N M R 2009 J. Phys.: Condens. Matter 21095501 Juan F, Cortijo A and Vozmediano M A H 2007 Phys. Rev. B 76165409

[11] Tapasztó L, Dobrik G, Nemes-Incze P, Vertesy G, Lambin Ph and Biró L P 2008 Phys. Rev. B 78233407

[12] Gibertini M, Singha A, Pellegrini V, Polini M, Vignale G, Pinczuk A, Pfeiffer L N and West K W 2009 Phys. Rev. B 79 241406(R)
[13] Park C-H, Yang L, Son Y-W, Cohen M L and Louie S G 2008 Nat. Phys. 4213

[14] Park C-H, Son Y-W, Yang L, Cohen M L and Louie S G 2008 Nano Lett. 82920

[15] Brey L and Fertig H A 2009 Phys. Rev. Lett. 103046809

[16] Ho J H, Chiu Y H, Tsai S J and Lin M F 2009 Phys. Rev. B 79115427

[17] Barbier M, Vasilopoulos P and Peeters F M 2010 Phil. Trans. R. Soc. A 3685499

[18] Chen X, Tao J-W and Ban Y 2011 Eur. Phys. J. B 70203 (arXiv:1004.0350v1 [cond-mat])

[19] Merzbacher E 1998 Quantum Mechanics (New York: Wiley)

[20] Pereira J M Jr, Vasilopoulos P and Peeters F M 2007 Appl. Phys. Lett. 90132122

[21] Wang L-G and Zhu S-Y 2010 Phys. Rev. B 81205444

[22] Barbier M, Vasilopoulos P and Peeters F M 2010 Phys. Rev. B 81075438 
